\title{
Des institutions dédiées à la formation à distance : passé ou futur de l'éducation?
}

\author{
Institutions dedicated to distance learning: past or future of \\ education?
}

\section{Instituciones dedicadas a la formación a distancia: ¿pasado o futuro de la educación?}

Cathia Papi, professeure

Université TÉLUQ, Canada

cathia.papi@teluq.ca

Viviane Glikman, enseignante-chercheuse

Membre du GEHFA (Groupe d'études - histoire de la formation des adultes), France

viviane.glikman@free.fr

Hélène Pulker, professeure

The Open University, Royaume-Uni

helene.pulker@open.ac.uk

\begin{abstract}
RÉSUMÉ
Cette introduction à la rubrique "Témoignages et entretiens " propose une lecture transversale des cinq entretiens qui la composent et présentent des institutions renommées dédiées à la formation à distance. Elle met en évidence le fait que ces institutions, malgré leurs différences, possèdent des points communs qui permettent de souligner des éléments essentiels dans l'histoire et l'actualité de la formation à distance. Trois de ces éléments sont ici abordés: la réponse à des besoins éducatifs et socioéconomiques, l'importance des évolutions technopédagogiques et les interrogations sur l'avenir d'institutions spécialisées face à la multiplication des acteurs et au large recours à la distance en période de crise sanitaire.
\end{abstract}

Mots-clés : formation à distance, institutions, universités, histoire, Canada, Royaume-Uni, Espagne, Suisse 
This introduction to the "Testimonies and interviews" offers a cross-analysis of the five interviews that constitute the section and presents well-known distance learning institutions. It highlights how these institutions, despite their differences, present similarities that underline essential elements in the history and current status of distance education. Three of these elements are discussed here: the response to educational and socio-economic needs, the importance of developments in learning design and the questioning of the future of specialized institutions in the light of the multiplication of actors and the widespread use of distance in times of a pandemic.

Keywords: distance education, institutions, universities, history, Canada, UK, Spain, Switzerland

\section{RESUMEN}

Esta introducción a la sección "Testimonios y entrevistas" propone una lectura transversal de las cinco entrevistas que la componen y que presentan a destacadas instituciones dedicadas a la formación a distancia. Cabe destacar que estas instituciones, a pesar de sus diferencias, tienen puntos en común que permiten subrayar elementos esenciales en la historia y en la situación actual de la formación a distancia. Tres de estos elementos se discuten aquí: la respuesta a necesidades educativas y socioeconómicas, la importancia de los avances tecnopedagógicos y las interrogaciones acerca del futuro de dichas instituciones especializadas frente a la multiplicación de los actores y la generalización de la educación a distancia en tiempos de crisis sanitaria.

Palabras clave: formación a distancia, instituciones, universidades, historia, Canadá, Reino Unido, España, Suiza

Les cinq entretiens présentés dans ce numéro confirment que la formation à distance s'est développée dans diverses institutions au cours du vingtième siècle, initialement sous forme de cours par correspondance, et perdure désormais sous forme d'enseignement en ligne. L'Open University britannique (OU), l'Université TÉLUQ (TÉLUQ), l'Athabasca University (AU) et l'Universitat Oberta de Catalunya (UOC) ont pour particularité d'être trois universités entièrement à distance, en contraste avec l'Unité Technologies de formation et apprentissage (TECFA) qui s'inscrit dans une université classique tout en favorisant l'émergence de dispositifs technopédagogiques innovants. Tandis que les trois premières, créées à la fin des années 1960 et au début des années 1970, ont connu le passage de l'enseignement par correspondance à la formation en ligne, l'UOC et l'unité TECFA, nées vingt ans plus tard, ont d'emblée intégré le numérique. La restitution des circonstances et de certains événements ayant marqué l'histoire de l'OU au Royaume-Uni, de la TÉLUQ au Québec, de l'AU au Canada anglais, de l'UOC en Espagne et de TECFA en Suisse permet ainsi de revenir sur l'évolution de la formation à distance au cours des dernières décennies. 
Nous aborderons ces entretiens à partir de quelques questions qui émergent d'une lecture transversale des récits recueillis. Pourquoi et comment ces institutions ont-elles été créées et ont-elles évolué? Quels ont été les défis et les obstacles rencontrés et comment ont-ils été surmontés? Quel a été le rôle des technologies dans les changements évoqués? Qu'en est-il de l'avenir de ces institutions depuis que la formation à distance n'est plus l'apanage de quelques établissements, mais une réalité à laquelle sont confrontés la plupart d'entre eux?

Nous insisterons ici sur quelques éléments de réponse à ces questions, souvent communs aux différents entretiens et qui nous semblent significatifs, espérant de la sorte inciter à lire ces entretiens et contribuer à ouvrir des pistes de réflexion permettant de faire le lien entre le passé et l'avenir de la formation à distance.

\section{L'essor de la formation à distance : une réponse à des besoins}

Comme nous le vivons depuis plus d'un an avec les mesures de confinement, qui ont fait de la formation à distance LA solution pour permettre une continuité dans l'éducation des élèves et des étudiants, celle-ci a toujours été initiée pour répondre à un besoin particulier. Ainsi, en France, l'établissement aujourd'hui connu sous le nom de Centre national d'enseignement à distance (CNED) a pour origine les bouleversements entraînés par la Seconde Guerre mondiale : il s'agissait alors de rendre possible la poursuite des études dans un contexte d'occupation et de déplacement de populations ${ }^{1}$. Les entretiens font, quant à eux, ressortir que trois des quatre universités intégralement à distance (OU, TÉLUQ, AU) sont nées de la nécessité et de la volonté de démocratiser l'accès à l'éducation à une époque où la demande en termes d'enseignements secondaire et supérieur augmentait rapidement. De son côté, l'UOC est venue répondre à un besoin d'ordre socioculturel et politique, celui de dispenser une formation à distance en langue catalane alors que l'Université nationale à distance espagnole (UNED) ${ }^{2}$ n'offrait que des cours en castillan. Ce renforcement de l'offre de formation supérieure visait donc à accueillir un plus grand nombre d'étudiants tout en évitant de longs déplacements aux personnes éloignées des campus et en permettant aux adultes actifs de se former indépendamment de leur emploi du temps professionnel ou familial. Ces spécificités en termes de distance, à la fois spatiale et temporelle, ont fait de la formation à distance une réponse potentiellement adaptée aux besoins.

Toutefois, les origines et les modes de fonctionnement des organisations ainsi créées sont variables. Toutes n'ont pas la même ancienneté et n'ont pas débuté avec les mêmes supports. Tandis que l'existence du CNED, précédemment mentionné, remonte aux années 1940 et s'est d'abord appuyée sur l'imprimé, l'OU, la TÉLUQ et l'AU sont apparues dans les années 1970 en recourant également à d'autres médias. L'unité TECFA, elle, n'a vu le jour qu'en 1989 et l'UOC au milieu des années 1990, au moment où le numérique s'installait durablement. Pendant que les gouvernements en place étaient demandeurs du déploiement d'une telle offre de formation dans le cas de l'OU et de l'AU, on peut noter que ce n'est pas le cas pour la TÉLUQ. Les sources de financement sont aussi différentes, de fonds publics aux droits payés par les étudiants pour les enseignements supérieurs de l'OU en passant par l'obtention de crédits obtenus dans le cadre d'appels d'offres pour TECFA.

\footnotetext{
1 Voir https://cursus.edu/actualites/43513/quelle-a-ete-la-crise-qui-a-donne-naissance-au-cned, site qui fait état d'un intéressant document abondamment illustré retraçant l'histoire du CNED : Bourrel, J.-R., Vidal, M., Mahieux, F. (2008). L'Histoire du CNED depuis 1939. Poitiers, France: CNED.

${ }^{2}$ Sur l'UNED, on peut se reporter à https://fr.wikipedia.org/wiki/Universit\%C3\%A9 nationale d\%27enseignement \%C3\%A0 distance et à son site (https://www.uned.es).
} 
On constate en outre que les changements gouvernementaux ont parfois influencé l'histoire de ces institutions, comme le montrent les débuts difficiles de l'AU, et qu'il faut du temps pour qu'elles soient pleinement reconnues (par le biais d'un mandat permanent, d'une lettre patente ou d'un statut de service public). De plus, au-delà des premières années, force est de constater que la vie des universités à distance est loin de suivre un long chemin tranquille, comme le prouvent notamment les nombreuses crises traversées par la TÉLUQ qui fut menacée de fermeture à plusieurs reprises.

Globalement, qu'il s'agisse d'institutions spécialisées dans la formation à distance ou d'unités comme TECFA, on constate aussi que la vision que les acteurs ont de ces organisations et de leurs missions contribue à en façonner le destin et à en pérenniser l'existence. Décisions politiques ou d'ordre privé et visions d'acteurs concourent donc, entre besoins socioéconomiques et idéaux éducatifs, à une adaptation des actions institutionnelles aux évolutions du contexte sociétal.

\section{Les évolutions technopédagogiques au cœur des institutions}

Deux points, en particulier, sont communs à ces institutions spécialisées en formation à distance. Le premier est d'impliquer une rupture temporelle et une division du travail entre la conception et la réalisation des cours, d'une part, et leur mise en œuvre de l'autre, notamment en ce qui concerne l'accompagnement et les activités collaboratives. Le second est d'intégrer les innovations technologiques susceptibles d'être mises au service tant de la création des cours que du tutorat. C'est ainsi que l'enseignement par correspondance s'est rapidement enrichi de l'audiovisuel, du multimédia, puis de la télématique (en France) et enfin d'Internet. Les cours imprimés, accompagnés d'émissions de radio ou de télévision, de disques ou de cassettes puis de cédéroms, ont donc progressivement été remplacés par des cours en ligne. Les courriers postaux et les échanges téléphoniques, même s'ils continuent d'exister, ont largement fait place aux communications par courriels ou visioconférences.

II est néanmoins nécessaire de se souvenir que, si le développement de la formation à distance s'est appuyé sur les innovations technologiques, il a également favorisé leur diffusion. L'unité TECFA et la TÉLUQ ont par exemple contribué au déploiement d'Internet en Suisse et au Québec. De fait, pour rendre l'éducation accessible au plus grand nombre, la formation à distance exige non seulement que les technologies soient disponibles, mais aussi qu'elles soient intégrées dans les foyers. On peut aussi noter que les changements technologiques relatifs aux moyens de production et de communication ont conduit les institutions spécialisées à ne pas se limiter aux recrutements classiques de personnels universitaires, mais également à s'adapter à l'entrée de nouveaux acteurs aux compétences liées aux technologies, aboutissant à remanier l'éventail des profils présents en leur sein et, par conséquent, à infléchir leur propre structure.

Par ailleurs, soutenues par l'avancée des recherches sur l'apprentissage, les modalités pédagogiques ont évolué et le modèle béhavioriste traditionnel a peu à peu cédé la place à des approches plus constructivistes. Dans le même temps, bien que la formation à distance s'adresse, au départ, davantage à des individus isolés qu'à des groupes, des tentatives pour mettre les étudiants en relation les uns avec les autres se sont fait jour, comme on le voit notamment à l'OU où la création de communautés est vivement encouragée. Convivialité et accompagnement sont ainsi présentés dans tous les entretiens comme des éléments essentiels pour permettre aux apprenants de suivre et réussir des études à distance.

Enfin, comme en témoignent en particulier les activités de l'unité TECFA et de l'Institut de technologie éducative de l'OU, l'importance des liens entre recherche et formation est souvent mise en évidence, liens qui permettent d'élaborer et de tester de nouveaux modèles de formation tout ou en partie en ligne, ainsi que d'améliorer les environnements d'apprentissage et le soutien offert aux apprenants. 


\section{Un avenir en train de s'écrire}

Si les institutions spécialisées en formation à distance ont le vent en poupe depuis le début de la pandémie et que leur expertise est souvent sollicitée pour aider les établissements habitués à ne délivrer que des cours en présentiel, force est de s'interroger sur leur avenir. De fait, au-delà des périodes actuelles de confinement, peut-on envisager que proposer des modalités d'enseignement à distance, hybride ou comodal, devienne la norme dans la plupart des organisations éducatives? Qu'en est-il, de plus, au temps où les MOOC ne sont pas seulement proposés par des organismes spécialisés dans la distance, à l'image de l'Open University ou de la TÉLUQ, mais peuvent l'être par toute équipe éducative désireuse de se faire connaître au-delà des murs de son lieu d'exercice?

Un tel élargissement du recours à la formation à distance, qui remet en cause le monopole des institutions pionnières dans ce domaine, pourrait sans doute réinterroger leur pertinence. Cependant, les entretiens font ressortir des spécificités qui confortent la légitimité et l'intérêt de ces institutions dans l'avenir. La première est certainement le public cible principalement constitué d'adultes actifs souhaitant autodiriger leurs apprentissages avec flexibilité et/ou ne possédant pas forcément les certifications requises pour entrer dans les universités traditionnelles, de telle sorte que l'OU et l'AU mettent en avant l'ouverture à tous comme la clé de l'avenir. Ces publics particuliers requièrent des rythmes, des démarches et des activités d'apprentissage conçus spécifiquement et dans une perspective d'individualisation, ce qui n'est souvent pas le cas des enseignements hybrides ou comodaux qui, bien qu'offrant une certaine flexibilité, s'adressent à des cohortes assujetties à un cheminement commun.

La seconde spécificité semble être l'expertise développée au cours de leur histoire par ces institutions, dont beaucoup conjuguent recherche et formation afin de déployer des dispositifs et des pratiques technopédagogiques efficaces et d'être (plus ou moins selon les lieux et les périodes) à la pointe de l'innovation dans ce domaine. En effet, la recherche et la mise en œuvre de nouvelles procédures prennent du temps et la crise sanitaire actuelle révèle les difficultés rencontrées par beaucoup d'enseignants et d'apprenants qui n'ont pas réellement fait l'expérience de la formation à distance, mais simplement de la mise en ligne de ressources ou de cours par visioconférences sans réel travail de conception pédagogique antérieur. Au Québec, certains syndicats disent ainsi qu'il ne s'agit pas d'enseignement à distance, mais d'enseignement en «mode non présentiel ». Ce constat est évidemment à double tranchant puisqu'il conforte la place des experts dans le domaine tout en dépréciant la formation à distance aux yeux de ceux qui n'en ont vécu qu'un ersatz dépourvu d'une conception pédagogique et d'une scénarisation adaptées, sans bénéficier de l'accompagnement et des interactions qui s'avèrent plus nécessaires que jamais en période de confinement où l'isolement ne se limite pas au seul contexte de formation. 